\title{
Evaluación y comparación de algoritmos de clasificación de eventos de tensión mediante su implementación en un DSP
}

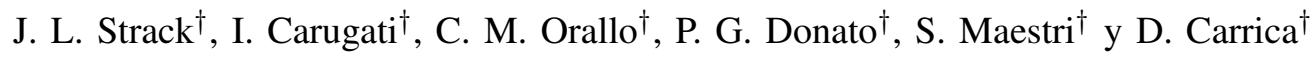 \\ $\dagger$ Instituto de Investigaciones Científicas y Tecnológicas en Electrónica (ICyTE), Universidad Nacional de \\ Mar del Plata, Consejo Nacional de Investigaciones Científicas y Técnicas (CONICET) \\ Av. Juan B. Justo 4302, Mar del Plata (7600), Buenos Aires, Argentina \\ $\dagger\{j l s t r a c k$, icarugati, orallo, donatopg, somaestri, carrica\}@fi.mdp.edu.ar
}

\begin{abstract}
This paper assesses the experimental implementation of three voltage events classification algorithms. They are known in the literature as symmetrical component algorithm, six-phase algorithm and space vector algorithm. They use the fundamental voltages and sequence's components estimated by a sequence detector proposed by the authors previously. This detector is synchronized with the network achieving phase and frequency adaptation and high rejection to the network disturbances. From implementation of classification algorithm and detector on a DSP (Digital Signal Processor), the performance of each algorithm in the classification of real events and their possible application in a PQM (Power Quality Monitor) was evaluated.
\end{abstract}

Resumen- Este trabajo evalúa la implementación experimental de tres algoritmos de clasificación de eventos de tensión. Los mismos son conocidos en la literatura como algoritmo de componentes simétricas, algoritmo de seis tensiones y algoritmo de vector espacial. Estos algoritmos utilizan las tensiones fundamentales $y$ las componentes de secuencia estimadas por un detector de secuencias propuesto por los autores en trabajos previos. Este detector se sincroniza con la red eléctrica, logrando adaptación en fase y frecuencia y provee de un gran rechazo a las perturbaciones de la red. A partir de la implementación de los algoritmos de clasificación y del detector en un DSP (Digital Signal Processor), se evaluó el desempeño de cada algoritmo en la clasificación de eventos reales y su posible aplicación en un instrumento medidor de calidad de la energía.

\section{INTRODUCCIÓN}

En la temática de calidad de la energía eléctrica, se denomina perturbaciones de tensión a las desviaciones de la tensión real respecto de su contraparte ideal, entendiendo a la tensión ideal como una forma de onda sinusoidal con amplitud y frecuencia constantes e iguales a sus valores nominales [1]. Uno de los tipos de perturbaciones más perjudiciales son los eventos, que se caracterizan por tener un inicio y fin bien demarcados, como los huecos de tensión (dips), sobretensiones (swells) e interrupciones. Si bien un hueco de tensión no es tan nocivo como una interrupción o una sobretensión, sucede con mayor frecuencia, por lo que el daño total originado por caídas de tensión, debidas principalmente a cortocircuitos, arranque de grandes motores y energización de transformadores, es mayor [2]. En consecuencia, la detección y clasificación de los mismos es de vital interés en la operación de sistemas eléctricos de potencia, puesto que el completo conocimiento de estos eventos permite determinar los tipos de fallas, la fuente de perturbación y su ubicación, entre otros; y actuar en consecuencia despejando la perturbación, mitigando sus efectos [3] o bien previniendo su nueva aparición.

En la literatura específica existen aportes teóricos sobre algoritmos de clasificación de eventos basados en herramientas heurísticas como Lógica Difusa, Redes Neuronales, Máquinas de Soporte Vectorial, Algoritmos Genéticos, entre otros [4], [5]. Si bien estos métodos han demostrado tener un buen desempeño en entornos de simulación, presentan un alto nivel de complejidad matemática, por lo cual su implementación en un procesador digital de señales resulta en un elevado requerimiento computacional.

Un enfoque diferente es el uso de algoritmos lógicos para clasificar eventos de tensión a partir del análisis de las componentes de secuencia [6], de las tensiones simples y compuestas [6] o de la trayectoria del vector espacial [7]. Este tipo de algoritmos, combinados con adecuadas técnicas de procesamiento digital de señales tienen un buen desempeño y requieren menos capacidad de procesamiento. Sin embargo, en la literatura específica [6]-[10] no abundan ejemplos de implementación en plataformas digitales, no se explica de manera consistente a través de que tipo de preprocesamiento se rechaza el ruido y el contenido armónico presentes en la red, ni son analizados rigurosamente bajo estas condiciones reales de operación.

En este trabajo se aborda la implementación de tres algoritmos presentados en la bibliografía, los cuales son implementados en un DSP junto a un detector de secuencias desarrollado por los autores y presentado en trabajos previos. Este último se basa en la mSDFT (del inglés modulated Sliding Discrete Fourier Transform) y en una técnica de período de muestreo variable (del inglés Variable Sampling Period Technique, VSPT), el cuál se adapta en fase y frecuencia y proporciona un rechazo elevado al contenido armónico y ruido presentes en la red [11].

Los algoritmos relevados se ensayaron con eventos reales capturados en laboratorio y luego sintetizados por un generador de señales programable. El objetivo es verificar si estos algoritmos son implementables en un DSP y si funcionan correctamente en la clasificación de eventos reales, ya que se pretende emplearlos en un monitor de calidad de la energía (del inglés Power Quality Monitor, PQM). 


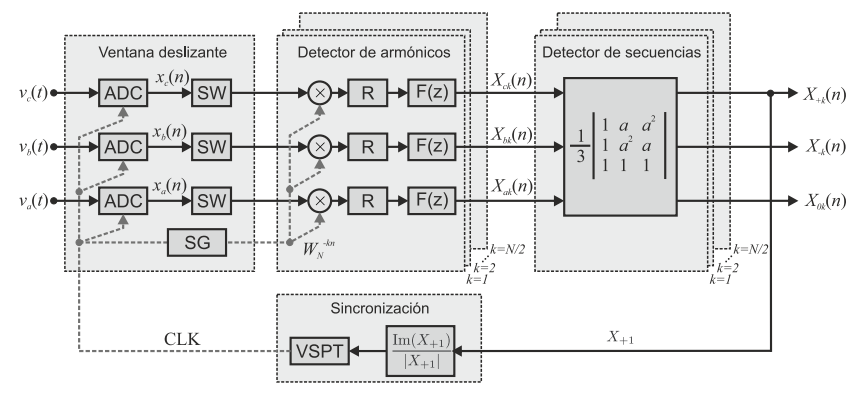

Fig. 1. Diagrama en bloques del detector de secuencias propuesto [11].

\section{PRe-filtro de LAS SEÑales de Tensión}

En [11], los autores han propuesto un medidor de armónicos y detector de secuencias para señales trifásicas basado en la mSDFT y en una técnica de período de muestreo variable, cuyo diagrama en bloques se observa en la Fig.1. La mSDFT es una implementación de la DFT (Discrete Fourier Transform) que utiliza la propiedad de modulación para calcular la k-ésima componente espectral de la señal en una ventana de tiempo deslizante de longitud $N$. La componente estimada por el algoritmo es un valor complejo y constante (la información de frecuencia es eliminada por la modulación), cuyo valor absoluto es la amplitud del armónico de interés y el argumento es la fase del mismo con respecto a la fase de la modulante. A diferencia de otras implementaciones de la DFT de ventana deslizante, la mSDFT es siempre estable y no acumula error, lo cual la hace muy adecuada para el diseño de sistemas de medición y monitoreo de las componentes armónicas de una determinada señal. En el trabajo citado, se propone el uso de una mSDFT por cada fase del sistema trifásico, empleándose la misma modulante para cada una de ellas. De esta forma, se estiman las tres k-ésimas componentes de la señal trifásica sin modificar la diferencia de fase original entre ellas. Luego, mediante el concepto de componentes simétricas, se estima la componente de secuencia positiva, negativa y cero de la misma. Debido a que la mSDFT sufre del problema típico de la DFT cuando la frecuencia fundamental $\left(f_{L}\right)$ de la señal es variable (efecto conocido como espectro esparcido), se utiliza una técnica de período de muestreo variable para ajustar dinámicamente la frecuencia de muestreo a $N \times f_{L}$. Como resultado del alto rechazo que provee la mSDFT a los armónicos de la red eléctrica y el ajuste dinámico del período de muestreo, el método propuesto en [11] presenta un excelente desempeño para el procesamiento de las tensiones y corrientes de línea en tiempo real. En el presente trabajo se va a utilizar este sistema como pre-filtro, estimando las componentes fundamentales de las tensiones de fase y sus componentes simétricas, que serán utilizadas por los métodos de clasificación de eventos.

\section{CRITERIOS DE CLASIFICACIÓN DE HUECOS DE TENSIÓN}

En la literatura se encuentran dos principales criterios de clasificación de huecos de tensión [12]: la clasificación de componentes simétricas (CS) y la clasificación ABC. La clasificación CS, basada en el análisis de las componentes simétricas de la tensión, distingue entre huecos con la
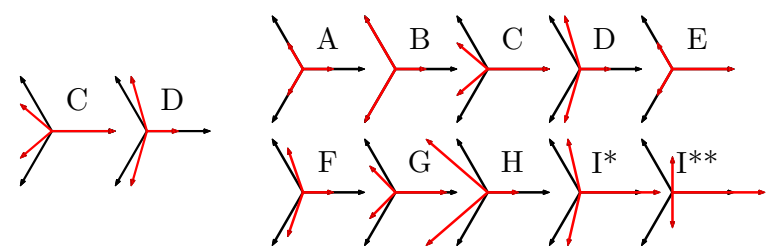

(a)
Fig. 2. Tipos de huecos de tensión: (a) criterio de clasificación CS, (b) criterio de clasificación $\mathrm{ABC}$.

principal caída de tensión en una fase (tipo D) y huecos con la principal caída de tensión en dos fases (tipo C), como se aprecia en la Fig. 2(a). Según este criterio, los huecos simétricos, es decir, con igual caída de tensión en las tres fases, son casos particulares de los dos anteriores. Por otro lado, la clasificación ABC distingue entre siete tipos de huecos (tipo A, ... ,tipo G) y tres tipos de huecos combinados con sobretensiones (tipo $\mathrm{H}$, tipo I* y tipo $\mathrm{I}^{* *}$ ), como se observa en la Fig. 2(b). Esta clasificación es más completa que la anterior y brinda más detalles a la hora de establecer una relación entre los huecos clasificados y los tipos de fallas que los causaron, por lo cual será la adoptada en el presente trabajo.

\section{Algoritmos de Clasificación de EVEntos}

En esta sección se presenta el principio de funcionamiento y las características principales de los algoritmos de clasificación de eventos evaluados.

\section{A. Algoritmo de Componentes Simétricas (ACS)}

Este algoritmo propuesto en [6] utiliza la siguiente expresión para la clasificación de eventos:

$$
T=\frac{1}{60^{\circ}} \arg \left\{\frac{V_{-1}}{1-V_{+1}}\right\}
$$

donde $T$ se redondea al entero más cercano y, $V_{-1}$ y $V_{+1}$ son las componentes de secuencia negativa y positiva de la tensión fundamental. A partir de este parámetro T que puede tomar valores enteros de 0 a 5 es posible determinar el tipo de hueco en la clasificación CS a partir de la Tabla I. Donde la letra $C$ ó $D$ indica el tipo de hueco y el subíndice $a, b$ o $c$ indica la fase afectada o no afectada según se trate de un hueco tipo $D$ o $C$ respectivamente.

Como se ha mencionado en la sección previa, la clasificación $\mathrm{ABC}$ resulta más conveniente para caracterizar huecos de tensión. Por lo tanto, para obtener el tipo de hueco de acuerdo al criterio $\mathrm{ABC}$, además del parámetro $T$, es necesario hallar tres variables adicionales: la tensión característica $V$ que representa la caída de tensión más significativa durante el hueco, el factor $F$ (definido como la relación entre la impedancia de secuencia positiva y negativa durante la falla), que es representativo del grado de desbalance del hueco [12], y la componente de secuencia

TABLA I

TIPO DE HUECO EN LA CLASIFICACIÓN CS DEL ACS.

\begin{tabular}{|c|c|c|c|c|c|c|}
\hline T & 0 & 1 & 2 & 3 & 4 & 5 \\
\hline Tipo CS & $C_{a}$ & $D_{c}$ & $C_{b}$ & $D_{a}$ & $C_{c}$ & $D_{b}$ \\
\hline
\end{tabular}


TABLA II

TIPO DE HUECO EN LA CLASIFICACIÓN CS DEL AST.

\begin{tabular}{|c|c|c|c|c|c|c|}
\hline Menor tensión & $V_{A 1}$ & $V_{B 1}$ & $V_{C 1}$ & $V_{B C 1}$ & $V_{C A 1}$ & $V_{A B 1}$ \\
\hline Tipo CS & $D_{a}$ & $D_{b}$ & $D_{c}$ & $C_{a}$ & $C_{b}$ & $C_{c}$ \\
\hline
\end{tabular}

cero fundamental $V_{01}$, las cuáles se obtienen a través de la siguiente expresión general [13]:

$$
\begin{aligned}
& V=V_{+1}-b^{6-T} V_{-1} \\
& F=V_{+1}+b^{6-T} V_{-1} \\
& V_{01}=\left|\dot{V}_{a 1}+\dot{V}_{b 1}+\dot{V}_{c 1}\right| / 3
\end{aligned}
$$

donde $b=\frac{1}{2}+\frac{1}{2} j \sqrt{3}$ es una rotación de $60^{\circ}$, y $\dot{V}_{a 1}, \dot{V}_{b 1}$ y $\dot{V}_{c 1}$ son los fasores de las tres tensiones de fase.

Finalmente, ingresando a la Tabla III con el parámetro $T$ (primera columna) y los valores de $V_{01}$ y $F$ se determina el tipo de hueco en la clasificación ABC y las fases afectadas.

\section{B. Algoritmo de seis tensiones (AST)}

Este algoritmo utiliza las tensiones eficaces fundamentales simples (3) y compuestas (4) desafectadas de la componente de secuencia cero fundamental [6], para clasificar huecos de tensión.

$$
\begin{gathered}
V_{A 1}=\left|\dot{V}_{a 1}-\dot{V}_{01}\right| \\
V_{B 1}=\left|\dot{V}_{b 1}-\dot{V}_{01}\right| \\
V_{C 1}=\left|\dot{V}_{c 1}-\dot{V}_{01}\right| \\
V_{A B 1}=\left|\dot{V}_{a 1}-\dot{V}_{b 1}\right| / \sqrt{3} \\
V_{B C 1}=\left|\dot{V}_{b 1}-\dot{V}_{c 1}\right| / \sqrt{3} \\
V_{C A 1}=\left|\dot{V}_{c 1}-\dot{V}_{a 1}\right| / \sqrt{3}
\end{gathered}
$$

El tipo de hueco de tensión, de acuerdo a la clasificación CS [12], es determinado a través de la tensión eficaz de menor valor, como se describe en la Tabla II.

$\mathrm{Al}$ igual que con el algoritmo anterior, interesa conocer el tipo de hueco de tensión de acuerdo a la clasificación ABC y las fases afectadas. Esto se logra, ingresando a la Tabla III con la menor tensión eficaz (segunda columna) y los valores de $V_{01}$ y $F$ que en este caso se calculan como:

$$
\begin{aligned}
& V=\min \left\{V_{A 1}, V_{B 1}, V_{C 1}, V_{A B 1}, V_{B C 1}, V_{C A 1}\right\} \\
& F=\max \left\{V_{A 1}, V_{B 1}, V_{C 1}, V_{A B 1}, V_{B C 1}, V_{C A 1}\right\}
\end{aligned}
$$

\section{Algoritmo de vector espacial (AVE)}

La transformación de vectores espaciales consiste en calcular un fasor (6), cuya magnitud y fase son representativas de la terna trifásica, reduciendo así la cantidad de información necesaria para representar a la misma.

$$
V_{e}(t)=\frac{2}{3}\left[v_{a}(t)+a v_{b}(t)+a^{2} v_{c}(t)\right]
$$

\begin{tabular}{|c|c|c|c|c|c|}
\hline $\mathbf{T}$ & Menor & $\mathbf{V}_{01}[\mathrm{pu}]$ & $\mathbf{F}[\mathbf{p u}]$ & Tipo & Fase \\
\hline $\begin{array}{l}3 \\
5 \\
1\end{array}$ & $\begin{array}{l}V_{A 1} \\
V_{B 1} \\
V_{C 1}\end{array}$ & $\geq 0.033$ & $>V \mathrm{y} \geq 0.967$ & $B$ & $\begin{array}{l}a \\
b \\
c\end{array}$ \\
\hline $\begin{array}{l}4 \\
0 \\
2\end{array}$ & $\begin{array}{l}V_{A B 1} \\
V_{B C 1} \\
V_{C A 1}\end{array}$ & $<0.033$ & $>V \mathrm{y} \geq 0.967$ & $C$ & $\begin{array}{lll}a & \mathrm{y} & b \\
b & \mathrm{y} & c \\
c \mathrm{y} & a\end{array}$ \\
\hline $\begin{array}{l}33 \\
5 \\
1\end{array}$ & $\begin{array}{l}V_{A 1} \\
V_{B 1} \\
V_{C 1}\end{array}$ & $<0.033$ & $>V \mathrm{y} \geq 0.967$ & $D$ & $\begin{array}{l}a \\
b \\
c\end{array}$ \\
\hline $\begin{array}{l}4 \\
0 \\
2\end{array}$ & $\begin{array}{l}V_{A B 1} \\
V_{B C 1} \\
V_{C A 1}\end{array}$ & $\geq 0.033$ & $>V \mathrm{y}<0.967$ & $E$ & $\begin{array}{lll}a & \text { y } & b \\
b & \text { y } & c \\
c & \text { y } & a\end{array}$ \\
\hline $\begin{array}{l}3 \\
5 \\
1\end{array}$ & $\begin{array}{l}V_{A 1} \\
V_{B 1} \\
V_{C 1}\end{array}$ & $<0.033$ & $>V \mathrm{y}<0.967$ & $F$ & $\begin{array}{l}a \\
b \\
c\end{array}$ \\
\hline $\begin{array}{l}4 \\
0 \\
2\end{array}$ & $\begin{array}{l}V_{A B 1} \\
V_{B C 1} \\
V_{C A 1}\end{array}$ & $<0.033$ & $>V \mathrm{y}<0.967$ & $G$ & $\begin{array}{lll}a & \mathrm{y} & b \\
b & \mathrm{y} & c \\
c \mathrm{y} & a\end{array}$ \\
\hline- & - & $<0.033$ & $\approx V \mathrm{y} \leq 0.9$ & $A$ & $a, b, c$ \\
\hline- & - & $<0.033$ & $\approx V \mathrm{y}>0.9$ & $E N$ & - \\
\hline
\end{tabular}

con $a=e^{j \frac{2 \pi}{3}}$ una rotación de $120^{\circ}$.

En [7], [8] se presenta una técnica para detectar, clasificar y caracterizar eventos de tensión, basada en el análisis de la trayectoria temporal del vector espacial. En un sistema trifásico sinusoidal simétrico las tres tensiones tienen la misma magnitud y están desfasadas $120^{\circ}$, por lo cual la
TABLA III

DETERMINACIÓN DEL TIPO DE HUECO ABC Y FASES PERTURBADAS A PARTIR DE LOS ALGORITMOS ACS Y AST.

trayectoria del vector espacial es una circunferencia de radio unitario. En [8] se demuestra que cuando ocurre un hueco de tensión simétrico la trayectoria es una circunferencia de radio proporcional a la profundidad del hueco, mientras que cuando ocurre un hueco de tensión asimétrico, la trayectoria es una elipse. A fin de distinguir los diferentes tipos de huecos y sobretensiones, se utilizan los parámetros de la elipse (7) y la tensión homopolar (2c).

$$
\begin{aligned}
& r_{m a}=\left|V_{+1}\right|+\left|V_{-1}\right| \\
& r_{m e}=|| V_{+1}|-| V_{-1}|| \\
& \phi_{i n c}=\frac{1}{2}\left(\phi_{+1}+\phi_{-1}\right) \\
& S I=\frac{r_{m e}}{r_{m a}}
\end{aligned}
$$

Donde $r_{m a}, r_{m e}, \phi_{i n c}$ y $S I$ son el radio mayor, radio menor, ángulo de inclinación e índice de forma, del ingles Shape Index, respectivamente. El criterio de clasificación se presenta en la Tabla IV, donde se observa que, a diferencia de los métodos anteriores, el AVE no sólo permite clasificar huecos de tensión, sino que también distingue huecos asociados con sobretensiones que afectan a una fase (tipo $\mathrm{H}$ ) o dos fases (tipo $\mathrm{I}^{*}$ e I**).

\section{ENSAYOS EXPERIMENTALES}

El detector de secuencias propuesto [11], y los tres algoritmos de clasificación de eventos presentados en las secciones previas fueron implementados en un DSP de punto flotante TMS320F28335 adoptándose un $N=128$ [muestras/ciclo].

En el diagrama de bloques de la Fig. 1 se ajustó $F(z)$ para que opere como un filtro de Butterworth pasabajos de segundo orden con una frecuencia de corte de $50 \mathrm{~Hz}$. El fin es filtrar y minimizar las oscilaciones en las tensiones fundamentales estimadas. Esto modifica levemente la dinámica, pero afecta favorablemente tanto a la estimación de las componentes de secuencias como al período de muestreo estimado, ya que interviene en el lazo de control que utiliza la parte imaginaria de la secuencia positiva. 
TABLA IV

DETERMINACIÓN DEL TIPO DE HUECO ABC Y FASES PERTURBADAS A PARTIR DEL ALGORITMO AVE.

\begin{tabular}{|c|c|c|c|c|c|}
\hline SI & $\phi_{\text {inc }}$ & $\mathbf{V}_{01}[\mathbf{p u}]$ & $\mathbf{r}_{\mathbf{m a}}[\mathbf{p u}]$ & Tipo & Fase \\
\hline$<1$ & $\begin{array}{c}90 \pm 15^{\circ} \\
-30 \pm 15^{\circ} \\
30 \pm 15^{\circ}\end{array}$ & $\geq 0.033$ & $\geq 0.967$ & $B$ & $\begin{array}{l}a \\
b \\
c\end{array}$ \\
\hline$<1$ & $\begin{array}{c}120 \pm 15^{\circ} \\
0 \pm 15^{\circ} \\
60 \pm 15^{\circ}\end{array}$ & $<0.033$ & $\geq 0.960$ & $C$ & $\begin{array}{lll}a & \mathrm{y} & b \\
b & \mathrm{y} & c \\
c & \mathrm{y} & a\end{array}$ \\
\hline$<1$ & $\begin{array}{c}90 \pm 15^{\circ} \\
-30 \pm 15^{\circ} \\
30 \pm 15^{\circ}\end{array}$ & $<0.033$ & $\geq 0.967$ & $D$ & $\begin{array}{l}a \\
b \\
c\end{array}$ \\
\hline$<1$ & $\begin{array}{c}120 \pm 15^{\circ} \\
0 \pm 15^{\circ} \\
60 \pm 15^{\circ}\end{array}$ & $\geq 0.033$ & $<0.967$ & $E$ & $\begin{array}{lll}a & \mathrm{y} & b \\
b & \mathrm{y} & c \\
c & \mathrm{y} & a\end{array}$ \\
\hline$<1$ & $\begin{array}{c}90 \pm 15^{\circ} \\
-30 \pm 15^{\circ} \\
30 \pm 15^{\circ}\end{array}$ & $<0.033$ & $<0.967$ & $F$ & $\begin{array}{l}a \\
b \\
c\end{array}$ \\
\hline$<1$ & $\begin{array}{c}120 \pm 15^{\circ} \\
0 \pm 15^{\circ} \\
60 \pm 15^{\circ} \\
\end{array}$ & $<0.033$ & $<0.960$ & $G$ & $\begin{array}{lll}a & \mathrm{y} & b \\
b & \mathrm{y} & c \\
c & \mathrm{y} & a\end{array}$ \\
\hline$<1$ & $\begin{array}{c}120 \pm 15^{\circ} \\
0 \pm 15^{\circ} \\
60 \pm 15^{\circ}\end{array}$ & $\geq 0.033$ & $\geq 0.967$ & $I^{* *}$ & $\begin{array}{lll}a & \mathrm{y} & b \\
b & \mathrm{y} & c \\
c & \mathrm{y} & a\end{array}$ \\
\hline SI & $\phi_{01}-\phi_{+1}$ & $\mathbf{V}_{01}[\mathbf{p u}]$ & - & Tipo & Fase \\
\hline$\approx 1$ & - & $<0.1$ & $\leq 0.9$ & $A$ & $a, b$ y $c$ \\
\hline$\approx 1$ & - & $<0.1$ & $>0.9$ & $E N$ & - \\
\hline$\approx 1$ & $\begin{array}{c}180 \pm 15^{\circ} \\
60 \pm 15^{\circ} \\
-60 \pm 15^{\circ}\end{array}$ & $\geq 0.1$ & - & $H$ & $\begin{array}{l}a \\
b \\
c \\
\end{array}$ \\
\hline$\approx 1$ & $\begin{array}{c}0 \pm 15^{\circ} \\
-120 \pm 15^{\circ} \\
120 \pm 15^{\circ}\end{array}$ & $\geq 0.1$ & - & $I^{*}$ & $\begin{array}{lll}a & \mathrm{y} & b \\
b & \mathrm{y} & c \\
c & \mathrm{y} & a\end{array}$ \\
\hline
\end{tabular}

Nota: tipo $E N$ corresponde a estado normal de la red.

Se ensayaron los tres algoritmos con tres eventos de tensión reales medidos en laboratorio y sintetizados por un generador de señales programable. A continuación se describen los tres ensayos y el desempeño de los algoritmos.

\section{A. Ensayo $n^{\circ}$ 1: hueco de tensión simétrico}

El primer evento ensayado consiste en un hueco tipo A que se caracteriza por presentar la misma caída de tensión en las tres fases. En la Fig. 3(a) se observan las señales adquiridas. El hueco de tensión es de aproximadamente 0.2 pu y 5.5 ciclos de duración y presenta un abrupto salto de fase tanto en su inicio como al finalizar el mismo. En la Fig. 3(b) se observan los módulos de las tensiones fundamentales y la frecuencia estimadas como ejemplo para evaluar el desempeño del pre-filtro. En la Fig. 3(c) se presenta la respuesta de los tres algoritmos de clasificación al implementar el detector de secuencias con el filtro de Butterworth incorporado. Mientras que en la Fig. 3(d) se presenta la respuesta de los tres algoritmos al quitar el filtro recién mencionado, a fin de ilustrar las mejoras producidas con la adición del mismo. En ambas figuras, para cada algoritmo se graficó a través de un osciloscopio de canales digitales un bus de 7 bits con el tipo de hueco detectado y otro bus de 7 bits que indica las fases mayormente afectadas. Para interpretar las dos salidas de cada algoritmo se debe tener en cuenta la codificación implementada que se describe en la Tabla V.

En el ensayo con filtro, Fig. 3(c), los tres algoritmos respondieron en menos de medio ciclo de red, siendo el más rápido el algoritmo de vector espacial (AVE), que a diferencia de los otros dos algoritmos, no presentó ningún

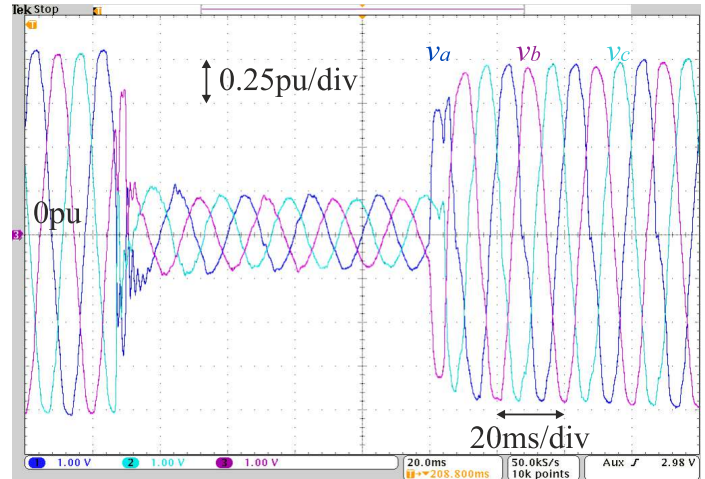

(a)

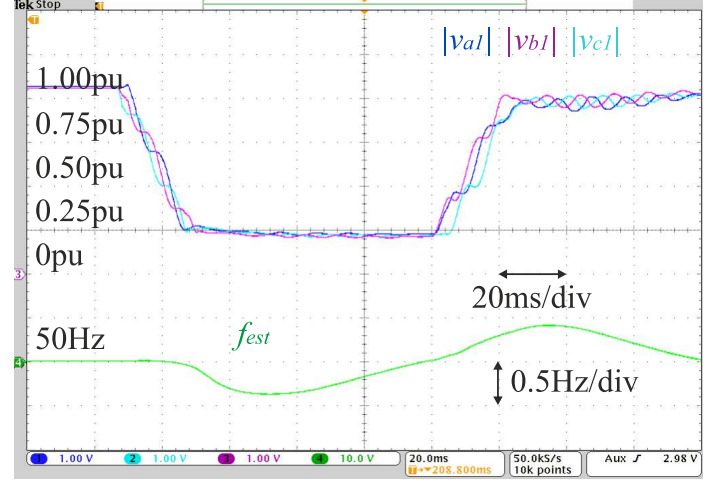

(b)

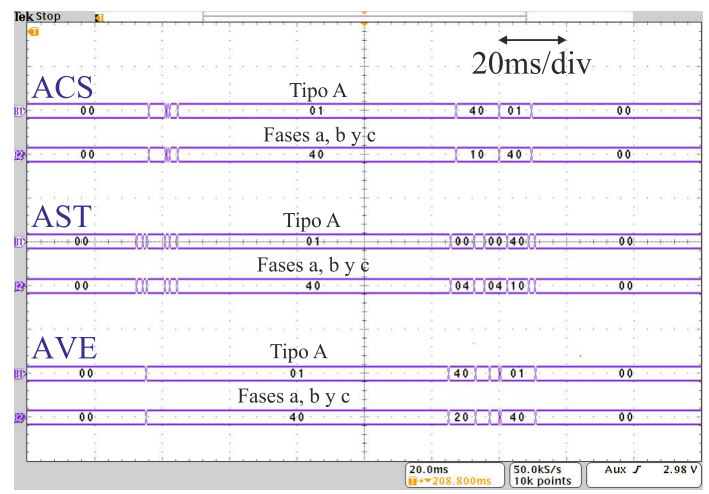

(c)

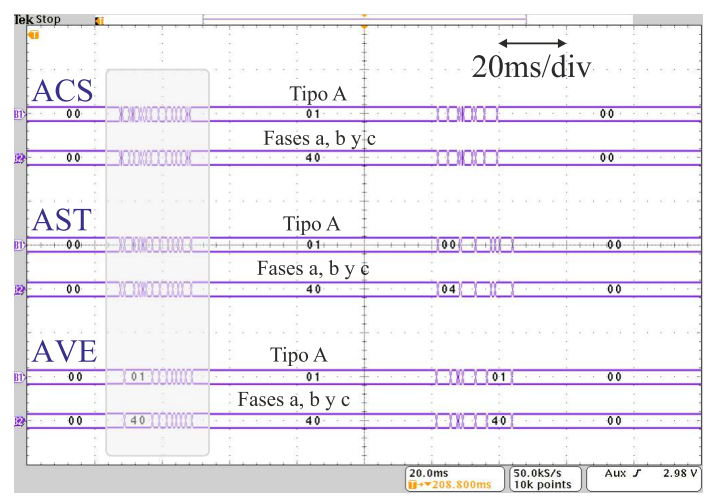

(d)

Fig. 3. Clasificación de un hueco de tensión simétrico. (a) Señales adquiridas, (b) componentes fundamentales y frecuencia estimadas por el detector de secuencias con filtro, (c) respuesta de los tres algoritmos con filtro y (d) respuesta de los tres algoritmos sin filtro.

transitorio en su respuesta al iniciarse el hueco de tensión. Mientras que, al finalizar el mismo, los tres algoritmos 
TABLA V

CODIFICACIÓN DE LAS SALIDAS DE LOS ALGORITMOS DE CLASIFICACIÓN DE EVENTOS PROGRAMADOS EN EL DSP

\begin{tabular}{|c|c||c|c|}
\hline $\begin{array}{c}\text { Valor del bus 1 } \\
\text { en hexadecimal }\end{array}$ & $\begin{array}{c}\text { Tipo } \\
\text { de hueco }\end{array}$ & $\begin{array}{c}\text { Valor del bus 2 } \\
\text { en hexadecimal }\end{array}$ & $\begin{array}{c}\text { Fases } \\
\text { afectadas }\end{array}$ \\
\hline 00 & $E N$ & 00 & ninguna \\
\hline 01 & $A$ & 01 & $a$ \\
\hline 02 & $B$ & 02 & $b$ \\
\hline 04 & $C$ & 04 & $c$ \\
\hline 08 & $D$ & 08 & $b$ y $c$ \\
\hline 10 & $E$ & 10 & $c$ y $a$ \\
\hline 20 & $F$ & 20 & $a$ у $c$ \\
\hline 40 & $G$ & 40 & $a, b$ у $c$ \\
\hline
\end{tabular}

Nota: tipo $E N$ corresponde a estado normal de la red.

presentaron estados transitorios, sin embargo, ninguno de estos superó un ciclo de duración. Por otro lado, en la configuración sin filtro, Fig. 3(d), se observa que los transitorios y saltos de fase durante el comienzo del hueco generan una gran cantidad de falsos disparos o transitorios en la salida de cada algoritmo, motivo por el cual, los tres métodos tardan un ciclo en clasificar correctamente y en forma sostenida el tipo de evento y las fases afectadas.

\section{B. Ensayo $n^{\circ}$ 2: hueco de tensión asimétrico en dos fases}

En segundo lugar, se ensayó un hueco asimétrico tipo $\mathrm{G}$ (Fig. 4) que afecta mayormente a las fases c y a, de aproximadamente $0.45 \mathrm{pu}$ y 4 ciclos de duración, que tiene una recuperación lenta desarrollando un hueco simétrico tipo A de amplitud superior a 0.83 pu durante aprox. 3 ciclos.

Se observa en la Fig. 4(c), que los tres algoritmos clasificaron en forma correcta ambos tipos de huecos y las fases mayormente afectadas. Los algoritmos ACS y AST tardaron menos de un ciclo en clasificar el primer evento y poco más de un ciclo en clasificar el segundo, mientras que el algoritmo AVE tardó menos de un ciclo en clasificar ambos eventos.

\section{Ensayo $n^{\circ}$ 3: hueco de tensión asimétrico en una fase}

Por último, se ensayó un hueco asimétrico tipo D (Fig. 5) que afecta mayormente a la fase c, de aproximadamente $0.26 \mathrm{pu}$ y 5 ciclos de duración. Se observa que en el transcurso del hueco, la fase c se encuentra además con una elevada distorsión armónica.

Se aprecia en la Fig. 5(c), que los tres algoritmos clasificaron en forma correcta el tipo de evento y la fase mayormente afectada. Los algoritmos ACS y AVE tardaron poco más de medio ciclo en clasificar el evento, mientras que el algoritmo AST tardó menos de medio ciclo. Los transitorios iniciales fueron de muy corta duración, ya que no hubo un salto de fase de gran magnitud que origine clasificaciones transitorias erróneas. Sin embargo, al finalizar el evento, los algoritmos ACS y AST pasaron de clasificar un hueco tipo $\mathrm{D}$ a un hueco tipo $\mathrm{F}$, que tiene características muy similares.

\section{Síntesis del desempeño de los algoritmos}

En la Tabla VI se sintetiza el desempeño de cada algoritmo en cada uno de los ensayos realizados. Se presenta la demora en clasificar el evento y la duración del mismo para cada algoritmo evaluado.

En [6] se muestra que el algoritmo ACS comete errores de clasificación cuando se presentan caídas de tensión y rotaciones de fase simétricas debido a variaciones en la carga, y
TABLA VI

SÍNTESIS DEL DESEMPEÑO DE CADA ALGORITMO.

\begin{tabular}{|c|c|c|c|}
\hline Ensayo & Algoritmo & $\begin{array}{c}\text { Demora } \\
\text { [ciclos] }\end{array}$ & $\begin{array}{c}\text { Duración } \\
\text { [ciclos] }\end{array}$ \\
\hline Ensayo no1 (con filtro) & ACS & 0.80 & 4.15 \\
Hueco tipo A - Fases a, b y c & AST & 0.80 & 4.05 \\
0.2pu - 5.5 ciclos & AVE & 0.40 & 4.50 \\
\cline { 2 - 4 } (sin filtro) & ACS & 1.00 & 3.65 \\
Hueco tipo A - Fases a, b y c & AST & 1.00 & 3.65 \\
0.2pu - 5.5 ciclos & AVE & 1.00 & 3.65 \\
\hline Ensayo no2 (con filtro) & ACS & 0.90 & 4.10 \\
Hueco tipo G - Fases c y a & AST & 0.90 & 4.45 \\
0.45pu - 4 ciclos & AVE & 0.95 & 4.15 \\
\cline { 2 - 4 } Hueco tipo A - Fases a, b y c & ACS & 1.15 & 2.15 \\
0.83pu - 3 ciclos & AST & 1.20 & 2.10 \\
& AVE & 0.95 & 2.35 \\
\hline Ensayo no3 (con filtro) & ACS & 0.50 & 3.55 \\
Hueco tipo D - Fase c & AST & 0.35 & 3.70 \\
0.26pu - 5 ciclos & AVE & 0.55 & 5.00 \\
\hline \multicolumn{2}{|l|}{}
\end{tabular}

que el algoritmo AST comete errores de clasificación cuando se presentan saltos de fase en las fases afectadas superiores a $30^{\circ}$ para un hueco en una fase y a $20^{\circ}$ para un hueco en dos fases. Estas limitaciones fueron estudiadas en [6] en forma analítica, sin considerar ningún prefiltro de las señales de tensión ni de las componentes simétricas. Cabe destacar que en las implementaciones realizadas en el presente trabajo se observa que el prefiltrado de dichas señales contribuye a la disminución de este tipo de fallas, puesto que la etapa de prefiltrado asegura adaptación en frecuencia y fase con la red, logrando así mayor robustez del sistema ante este tipo de perturbaciones.

\section{CONCLUSIONES}

Se implementaron tres algoritmos de clasificación de eventos de tensión en un DSP y se evaluaron con eventos capturados en laboratorio y luego sintetizados. El desempeño de los tres métodos cuyas variables de entrada son estimadas y actualizadas en cada muestra por el detector de secuencias propuesto por los autores en [11], es adecuado para fines prácticos, teniendo en cuenta que las señales adquiridas provienen de mediciones en la red eléctrica, es decir, contienen ruido, armónicos, transitorios y saltos de fase. Esto se debe a que la mSDFT asociada con la VSPT se ajusta en frecuencia y fase, y provee gran rechazo al contenido armónico y ruido de alta frecuencia. No obstante, se comprobó que la adición de un filtro de Butterworth mejora sustancialmente la respuesta de los clasificadores, ya que suaviza los transitorios en las tensiones y componentes de secuencia y en el propio período de muestreo estimado, debido a que interviene en el lazo de control, el cual toma la parte imaginaria de la secuencia positiva. Si bien los tres algoritmos tuvieron un desempeño adecuado, el AVE se destaca por clasificar los eventos en forma rápida $\mathrm{y}$ durante un tiempo muy cercano a la duración del mismo, y porque permite clasificar sobretensiones además de huecos de tensión, por lo cual resulta de interés su aplicación en un monitor de calidad de la energía.

\section{AGRADECIMIENTOS}

Los autores agradecen al Instituto de Investigaciones Científicas y Tecnológicas en Electrónica (ICyTE) de la UNMdP, al CONICET, a la ANPCyT, y al MinCyT por el apoyo al presente trabajo. 


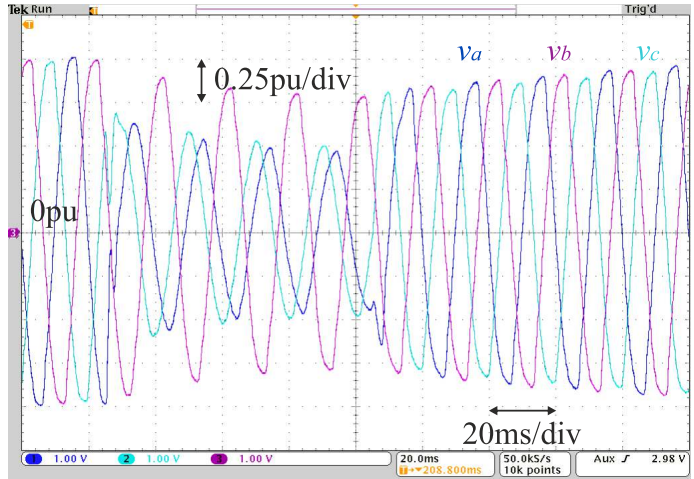

(a)

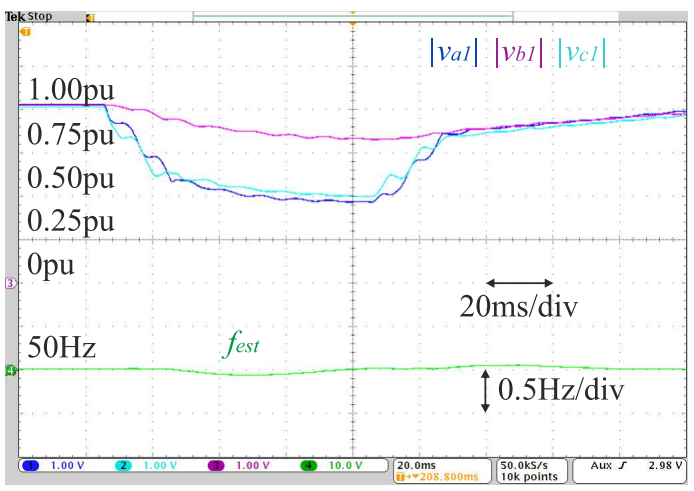

(b)

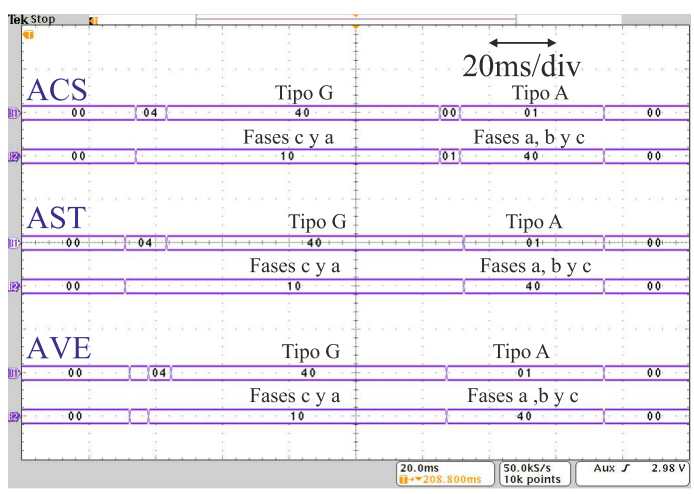

(c)

Fig. 4. Clasificación de un hueco asimétrico que afecta a dos fases (a) Señales adquiridas, (b) tensiones fundamentales y frecuencia estimadas por el detector de secuencias y (c) respuesta de los algoritmos con filtro.

\section{REFERENCIAS}

[1] M. H. J. Bollen, Understanding Power Quality Problems: Voltage Sags and Interruptions. Wiley - IEEE Press, 1999.

[2] L. L. Grigsby, Electric Power Generation, Transmission, and Distribution, 3rd ed. CRC Press, 2012.

[3] O. Ipinnimo, S. Chowdhury, S. Chowdhury, and J. Mitra, "A review of voltage dip mitigation techniques with distributed generation in electricity networks," Electric Power Systems Research, vol. 103, pp. $28-36,2013$.

[4] O. P. Mahela, A. G. Shaik, and N. Gupta, "A critical review of detection and classification of power quality events," Renewable and Sustainable Energy Reviews, vol. 41, pp. 495 - 505, 2015.

[5] D. Granados-Lieberman, R. j. Romero-Troncoso, R. a. Osornio-Rios, A. Garcia-Perez, and E. Cabal-Yepez, "Techniques and methodologies for power quality analysis and disturbances classification in power systems: a review," IET Generation, Transmission Distribution, vol. 5, no. 4, pp. 519-529, April 2011.

[6] M. H. J. Bollen, "Algorithms for characterizing measured three-phase unbalanced voltage dips," IEEE Transactions on Power Delivery, vol. 18, no. 3, pp. 937-944, July 2003.

[7] V. Ignatova, P. Granjon, S. Bacha, and F. Dumas, "Classification and

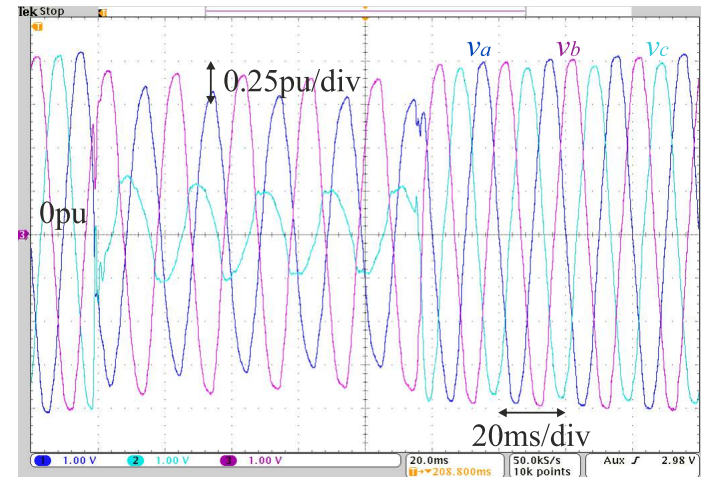

(a)

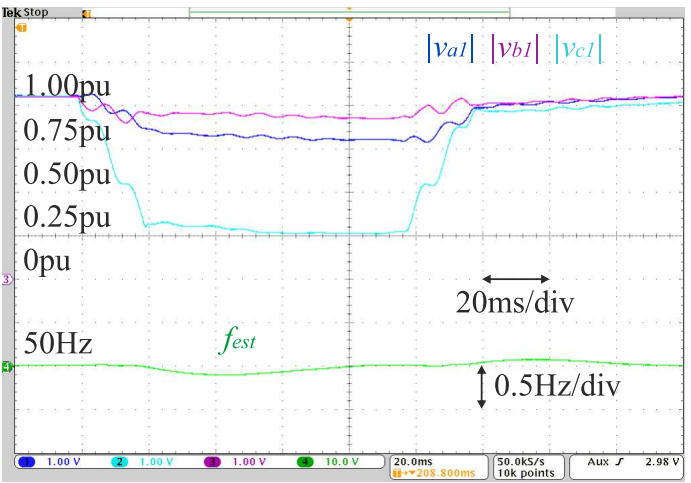

(b)

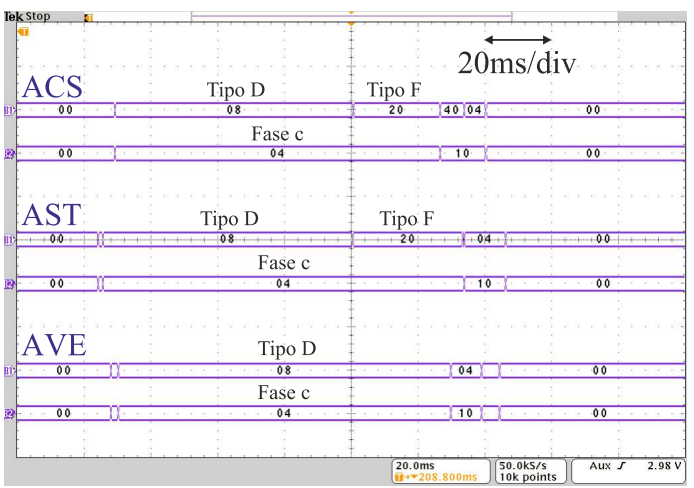

(c)

Fig. 5. Clasificación de un hueco asimétrico que afecta a una fase. (a) Señales adquiridas, (b) tensiones fundamentales y frecuencia estimadas por el detector de secuencias y (c) respuesta de los algoritmos con filtro.

characterization of three phase voltage dips by space vector methodology," in Future Power Systems, 2005 International Conference on, Nov 2005, pp. 6 pp. -6

[8] V. Ignatova, P. Granjon, and S. Bacha, "Space vector method for voltage dips and swells analysis," IEEE Transactions on Power Delivery, vol. 24, no. 4, pp. 2054-2061, Oct 2009.

[9] M. H. Chia and A. M. Khambadkone, "Subcycle voltage dip classification using matrix pencil method with ellipse fitting algorithm," IEEE Transactions on Industry Applications, vol. 51, no. 2, pp. 16601668, March 2015.

[10] M. R. Alam, K. M. Muttaqi, and A. Bouzerdoum, "Characterizing voltage sags and swells using three-phase voltage ellipse parameters," IEEE Transactions on Industry Applications, vol. 51, no. 4, pp. 2780 2790, July 2015.

[11] I. Carugati, C. M. Orallo, P. G. Donato, S. Maestri, and D. Carrica, "Three-phase harmonics measurement method based on msdft," IEEE Latin America Transactions, vol. 12, no. 7, pp. 1250-1257, Oct 2014.

[12] M. H. J. Bollen and L. D. Zhang, "Different methods for classification of three-phase unbalanced voltage dips due to faults," Electric Power Systems Research, vol. 66, no. 1, pp. 59-69, 2003.

[13] M. H. J. Bollen and I. Y.-H. Gu, Signal Processing of Power Quality Disturbances. Wiley - IEEE Press, 2006. 